Gynäkologe 2018·51:922-925

https://doi.org/10.1007/s00129-018-4352-z

(C) Springer Medizin Verlag GmbH, ein Teil von Springer Nature 2018

CrossMark

\author{
Ricardo E. Felberbaum ${ }^{1} \cdot$ Tanja Fehm ${ }^{2} \cdot$ Klaus Diedrich $^{3}$ \\ 'Klinik für Frauenheilkunde und Geburtshilfe, Klinikum Kempten, Klinikverbund Kempten - Oberallgäu, \\ Kempten, Deutschland \\ ${ }^{2}$ Universitätsfrauenklinik Düsseldorf, Düsseldorf, Deutschland \\ ${ }^{3}$ Groß Sarau, Deutschland
}

\title{
Karzinome und Fertilität
}

Die maßgeblichen, in den letzten Jahrzehnten zu beobachtenden Fortschritte in Prävention, Früherkennung, Diagnostik und Therapie onkologischer Erkrankungen haben dazu geführt, dass eine immer größere Zahl, vor allem auch jüngerer Patientinnen und Patienten den Krankheitszustand überstehen und als langfristig geheilt betrachtet werden können. Diese Gruppe der im angloamerikanischen Sprachraum „cancer survivors“ genannten Menschen stellt mit Recht den Anspruch auf die Wiederherstellung und den Wiedergewinn eines normalen Lebens. Eine immer wichtigere Rolle spielt dabei die Möglichkeit, eigene leibliche Kinder zu bekommen und eine normale Familie gründen zu können bzw. zumindest der Erhalt der Option darauf.

》) "Cancer survivors" stellen zurecht den Anspruch auf den Wiedergewinn eines normalen Lebens

Es ist daher nur konsequent, dass die amerikanische klinische Krebsgesellschaft (American Society of Clinical Oncology, ASCO) im Jahr 2018 bereits die zweiten aktualisierten, sehr ausführlichen Leitlinien zur Fertilitätsprotektion bei onkologischen Erkrankungen publiziert hat. Ihr Zweck besteht in der Bereitstellung aktueller Empfehlungen für die Anwendung adäquater Methoden zur Fertilitätserhaltung für krebskranke Erwachsene und auch Kinder. Dabei sollte das Gespräch über die Möglichkeit einer nachfolgenden Unfruchtbarkeit mit Krebspatientinnen und -patienten, die sich in der reproduktiven Lebens- phase befinden, oder auch mit Eltern bzw. Erziehungsberechtigten möglichst früh stattfinden. Von ärztlicher Seite sollte die Bereitschaft signalisiert werden, Optionen zur Fertilitätserhaltung $\mathrm{zu}$ diskutieren und die Betroffenen vor Beginn der onkologischen Therapie auch zur Beratung noch an ein reproduktionsmedizinisches Zentrum zu verweisen. Obwohl sich jede Patientin und jeder Patient zunächst auf die Krebsdiagnose konzentriert und auch konzentrieren sollte, wird das Ausbleiben eines solchen Gesprächs, das Verschweigen oder das Ignorieren einer möglichen nachfolgenden Unfruchtbarkeit als großes Versäumnis der Ärzte und Ärztinnen von den Betroffenen beklagt.

Spermien-, Oozyten- und Embryokryokonservierung gelten als Standardpraxis und sind weit verbreitet. Während die Spermiengewinnung zur anschließenden Kryokonservierung im Regelfall problemlos gelingt, stellen die Möglichkeiten zum Fertilitätserhalt bei der Frau eine größere Herausforderung dar. Für Frauen, die an Krebs erkranken, gibt es mehrere Optionen zur Fertilitätserhaltung, und diese könnten je nach Alter der Patientin, dem Risiko einer Beteiligung der Eierstöcke, der verfügbaren Zeit und der Art des Krebses mit unterschiedlichen Vor- und Nachteilen einhergehen. Zwar gibt es mehrere Möglichkeiten, doch zurzeit sind die Kryokonservierung von unbefruchteten Eizellen, Eizellen im Vorkernstadium und Embryonen oder die Kryokonservierung von Eierstockgewebe die zuverlässigsten Methoden.

Hinsichtlich der Kryokonservierung von unbefruchteten Eizellen, Eizellen im Vorkernstadium oder auch von Embryonen muss berücksichtigt werden, dass für 
Hier steht eine Anzeige.

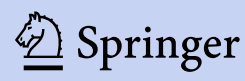


die postpubertäre Frau ein noch passendes Zeitfenster bis zum Beginn der geplanten gonadotoxischen Behandlung existieren muss, da die ovarielle Stimulation als Grundvoraussetzung für die Gewinnung einer ausreichenden Zahl fertilisierbarer Eizellen einen Zeitraum von 10-14 Tagen benötigt. Dank moderner Protokolle kann die Stimulation an jedem Zyklustag gestartet werden („random start"). Im Durchschnitt können dabei bei einer 30- bis 36-jährigen Frau ca. 10-13 befruchtungsfähige Eizellen in der Metaphase II gewonnen werden. Mit zunehmendem Alter nimmt diese Zahl aber sukzessive ab und mindert dadurch die Hoffnung auf einen erfolgreichen Fertilitätserhalt.

Im Unterschied dazu ist die Kryokonservierung von Ovarialgewebe für die spätere Retransplantation bei Kinderwunsch und therapieinduziertem vorzeitigem Ovarialversagen eine $\mathrm{Me}$ thode, die innerhalb kürzester Zeit, also innerhalb von wenigen Tagen durchgeführt werden kann. Die Entnahme des Ovarialgewebes auf laparoskopischem Wege erfolgt zyklusunabhängig, wodurch eine Verzögerung der onkologischen Therapie nicht zu erwarten ist. Die Schwangerschaftsraten nach Retransplantation von Ovarialgewebe bei Frauen, die zum Zeitpunkt der Kryokonservierung unter 35 Jahre alt waren, liegen bei $33 \%$, im Vergleich zu nur $18 \%$ bei Frauen, die zum Zeitpunkt der Entnahme älter als 35 Jahre alt waren. Bei Frauen über 40 Jahren scheint die Maßnahme nicht erfolgreich zu sein.

Die Debatte, ob Gonadotropin-releasing-Hormon-Agonisten (GnRHa) zur Unterdrückung der Ovarialfunktion wirksam sind im Sinne der Erhaltung der Fruchtbarkeit vor zytotoxischer Therapie ist sicherlich noch nicht beendet. Neueste Metaanalysen aber weisen auf einen solchen klinisch relevanten Effekt hin, sodass die GnRHa-Behandlung als rasch applizierbare, nebenwirkungsarme Begleittherapie Patientinnen in der Hoffnung auf eine Verringerung der Wahrscheinlichkeit einer chemotherapieinduzierten Ovarialinsuffizienz angeboten werden sollte, wenn andere, effizientere Fertilitätserhaltungsmethoden nicht durchführbar sind, sowie bei jungen Frauen mit Brustkrebs. In der aktuellsten Metaanalyse betrug die Rate an chemotherapieinduzierter Ovarialinsuffizienz nur 14,1\% in der GnRHa-Gruppe und $30,9 \%$ in der Kontrollgruppe. Es konnten keine signifikanten Unterschiede im krankheitsfreien Überleben und Gesamtüberleben zwischen den beiden Gruppen beobachtet werden. Allerdings sollten die GnRHa nicht anstelle von bewährteren Methoden zur Fertilitätserhaltung verwendet werden. Dies gilt vor allem angesichts der Tatsache, dass der Bereich der Kryokonservierung von Eierstockgewebe rasch voranschreitet und sich in Zukunft zu einer Standardtherapie entwickeln könnte.

In den letzten Jahren wurde dank der Verbesserung der Prognose von Brustkrebspatientinnen zunehmend Aufmerksamkeit auf die Fruchtbarkeitsproblematik der jüngeren betroffenen Frauen fokussiert. Dabei stellt dieses in der Mehrzahl der Fälle hormonresponsive Karzinom sicher einen Sonderfall dar. Für junge Frauen im reproduktionsfähigen Alter bedeutet dies, dass jeder Patientin mit der Diagnose Brustkrebs eine ausführliche Beratung hinsichtlich der Möglichkeiten fertilitätsprotektiver Maßnahmen angeboten werden sollte, umso mehr, wenn eine systemische Chemotherapie indiziert ist. Die protektiven möglichen Maßnahmen reichen von der ovariellen Funktionssuppression über die Kryokonservierung von Eizellen (unbefruchtet oder befruchtet) bis hin zur operativen Gewinnung von Ovarialgewebe, dessen Kryokonservierung und späteren Retransplantation. Tatsächlich gestattet aber die Brustkrebserkrankung in fast jedem Fall das notwendige Zeitfenster, um eine kontrollierte ovarielle Hyperstimulation zur Gewinnung von Eizellen zu ermöglichen. Die Brustkrebspatientin ist die Patientin, bei der die Asservierung und Kryokonservierung von Gameten, imprägnierten Eizellen oder auch von Embryonen als Mittel der ersten Wahl erscheint, um auf diesem Wege die Fertilität zu erhalten. Für die kontrollierte ovarielle Stimulation bei Brustkrebspatientinnen mit hormonsensiblem Karzinom sind Protokolle unter Begleitmedikation mit Tamoxifen oder Aromatasehemmer entwickelt worden.
Entsprechend den bisher verfügbaren Daten scheinen diese kein Sicherheitsrisiko darzustellen. Sowohl im klassischen, „langen Protokoll“ unter Verwendung von GnRHa als auch im „multiple dose GnRH-antagonist protocol“ konnten unter Begleitmedikation mit Tamoxifen zwischen 8 und 13 Eizellen gewonnen werden. Alternativ zur Rezeptorblockade mit Tamoxifen erscheint die Anwendung von Aromatasehemmern als Begleitmedikation zur kontrollierten ovariellen Hyperstimulation attraktiv. Mit dieser Art der Behandlung wird die Östradiolsekretion signifikant vermindert. In der Situation einer neu festgestellten Brustkrebserkrankung, einer geplanten neoadjuvanten Chemotherapie oder auch adjuvanten Therapie erscheint das Warten auf den Einsatz der Menstruation aus onkologischer Sicht immer fragwürdig. Es konnte aber mittlerweile gezeigt werden, dass die kontrollierte ovarielle Hyperstimulation $\mathrm{zu}$ jedem Zeitpunkt des Zyklus begonnen werden kann („random start").

\section{》) Nicht immer werden sich die geweckten Hoffnungen auch wirklich erfüllen lassen}

In Deutschland hat sich ein funktionierendes, das gesamte Bundesgebiet überspannendes Netzwerk gebildet (FertiPROTEKT), das allen Betroffenen die Möglichkeit zur Wahrnehmung fertilitätsprotektiver Maßnahmen geben soll. Mittlerweile umfasst dieses Netzwerk auch Zentren aus Österreich und der Schweiz (www.fertiprotekt.de, www.fertiprotekt.ch, www.fertiprotekt. at, www.fertiprotekt.com). Seit 2008 können auch private Zentren Mitglieder werden. Da das Netzwerk trotz national und international hohem Bekanntheitsgrad, z. B. in der Zusammenarbeit mit anderen Fachgesellschaften, bei der Erarbeitung von Leitlinien, Diskussionen mit Krankenkassen etc., keine anerkannte Rechtsform darstellte, wurde 2015 beschlossen, es in einen eingetragenen Verein zu überführen: FertiPROTEKT Netzwerk e.V. wurde am 10. November 2015 in Hamburg gegründet. Seine Geschäftsstelle befindet sich in Marburg. 
Kürzlich erschienen ist die S2k-Leitlinie der AWMF (Arbeitsgemeinschaft der Wissenschaftlichen Medizinischen Fachgesellschaften) „Fertilitätserhalt bei onkologischen Erkrankungen“, an der FertiPROTEKT-Mitglieder intensiv mitgearbeitet haben.

Zusammenfassend lässt sich sagen, dass der Verlust der Fertilität nach erfolgter Behandlung eines Malignoms zweifelsfrei einen enormen Einfluss auf die Lebensqualität der Betroffenen hat. So ist es nicht verwunderlich, dass bis zu $75 \%$ aller jungen Frauen, die mit der Diagnose Krebs konfrontiert sind, ihre Fertilität in Gefahr sehen. Über das Gefährdungspotenzial gut informiert $\mathrm{zu}$ sein, ist von großer Bedeutung, um Entscheidungen treffen zu können und um Autonomie zurückzuerlangen im Angesicht möglicher gonadotoxischer Konsequenzen anstehender Behandlungsmaßnahmen und damit drastisch veränderter Zukunftsaussichten. Dennoch wird es nicht in jedem Fall gelingen, die geweckten Hoffnungen auch wirklich im weiteren Leben der Patientinnen und Patienten zu erfüllen. Dies gilt in besonderem Maße für das Ovarialkarzinom, wo allein der Borderline-Tumor Optionen zum Fertilitätserhalt ermöglicht. Daher ist es wichtig, in allen Fällen von Anfang an zu vermitteln, dass Elternschaft nicht der einzige Sinn des Lebens sein kann und darf.

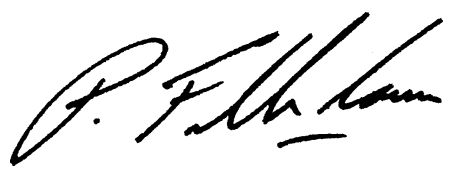

Prof. Dr. med. Ricardo E. Felberbaum

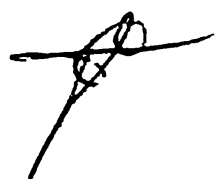

Prof. Dr. med. Tanja Fehm

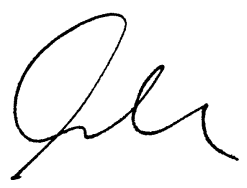

Prof. Dr. med. Dr. h.c. mult. Klaus Diedrich

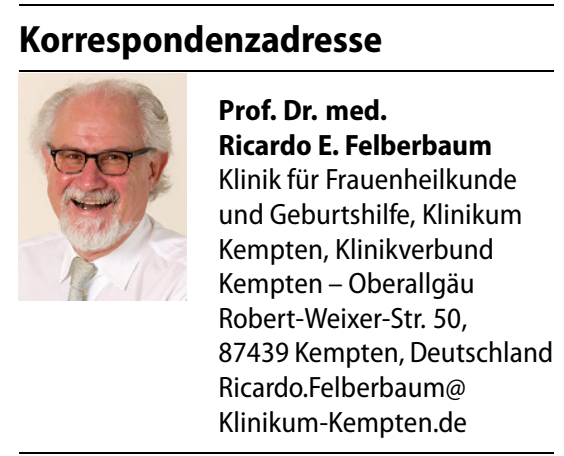

Interessenkonflikt. R.E. Felberbaum, T. Fehm und K. Diedrich geben an, dass kein Interessenkonflikt besteht.

\section{Ausschreibung SMG-Wissen- schaftspreis 2019}

Der Schweizerischen Menopausengesellschaft ist es ein großes Anliegen, Forschung auf dem Gebiet der Menopause bzw. Frauengesundheit in der 2. Lebenshälfte zu fördern. Hierfür wird der Wissenschaftspreis über 1000,00 CHF für das beste Abstract verliehen.

Die SMG fordert junge wie auch erfahrene Wissenschaftler auf, ihre Arbeit einzureichen und sie einem fachkundigen Kreis vorzustellen, miteinander zu diskutieren und gegenseitig voneinander zu profitieren.

Besonderer Wert wird auf die wissenschaftliche Bearbeitung der Fragestellungen, die sich fachübergreifend aus zahlreichen Bereichen wie Diabetologie, Ernährungswissenschaft, Innere Medizin, Kardiologie, Onkologie, Ophthalmologie, Osteologie etc. ergeben, und auf die Förderung des interdisziplinären Dialogs gelegt.

Einreichungen können aus Deutschland, Österreich oder der Schweiz in deutscher oder englischer Sprache erfolgen. Das Abstract sollte wie folgt gegliedert sein:

- Einleitung

- Material und Methoden

- Ergebnisse

- Diskussion

- Schlussfolgerung

Die maximale Wortzahl beträgt 400, einzureichen als Word-Dokument.

Die Einreichung der Abstracts sollte bitte über E-Mail: administration@menopause.ch mit dem Betreff: „SMG-Neue Wissenschaft" erfolgen und alle Kontaktdaten des Einreichers enthalten.

Der Gewinner/die Gewinnerin wird sein/ihr Abstract in einem 10-minütigen freien Vortrag beim Women's Health Congress am 10.1.2019 in Bern präsentieren.

Deadline für die Einreichung ist der 1.12.2018. 